\title{
Communication
}

\section{Programmed co-assembly of one-dimensional binary superstructures by liquid soft confinement}

\author{
Dan Guo, Yanan Li, Xu Zheng, Fengyu Li, Shuoran Chen, \\ Mingzhu Li, Qiang Yang, Huizeng Li, and Yanlin Song \\ J. Am. Chem. Soc., Just Accepted Manuscript • DOI: 10.1021/jacs.7b09738 • Publication Date (Web): 19 Dec 2017 \\ Downloaded from http://pubs.acs.org on December 19, 2017
}

\section{Just Accepted}

"Just Accepted" manuscripts have been peer-reviewed and accepted for publication. They are posted online prior to technical editing, formatting for publication and author proofing. The American Chemical Society provides "Just Accepted" as a free service to the research community to expedite the dissemination of scientific material as soon as possible after acceptance. "Just Accepted" manuscripts appear in full in PDF format accompanied by an HTML abstract. "Just Accepted" manuscripts have been fully peer reviewed, but should not be considered the official version of record. They are accessible to all readers and citable by the Digital Object Identifier (DOI®). "Just Accepted" is an optional service offered to authors. Therefore, the "Just Accepted" Web site may not include all articles that will be published in the journal. After a manuscript is technically edited and formatted, it will be removed from the "Just Accepted" Web site and published as an ASAP article. Note that technical editing may introduce minor changes to the manuscript text and/or graphics which could affect content, and all legal disclaimers and ethical guidelines that apply to the journal pertain. ACS cannot be held responsible for errors or consequences arising from the use of information contained in these "Just Accepted" manuscripts. 


\title{
Programmed Co-assembly of One-dimensional Binary Superstruc- tures by Liquid Soft Confinement
}

\author{
Dan Guo, ${ }^{\dagger, \downarrow}$ Yanan $\mathrm{Li}^{\dagger,+\neq}$ Xu Zheng, ${ }^{\dagger}$ Fengyu Li, ${ }^{\dagger}$ Shuoran Chen, ${ }^{\S}$ Mingzhu Li, ${ }^{\dagger}$ Qiang Yang, ${ }^{\dagger,+}$ Huizeng \\ $\mathrm{Li}^{\dagger, *}$ and Yanlin Song ${ }^{, \dagger}$ \\ ${ }^{\dagger}$ Key Laboratory of Green Printing, Institute of Chemistry Chinese Academy of Sciences, Beijing 100190, P. R. China; Bei- \\ jing Engineering Research Center of Nanomaterials for Green Printing Technology, Beijing National Laboratory for Molecu- \\ lar Sciences, Beijing 100190, P. R. China; ${ }^{*}$ Department of Chemistry, University of Chinese Academy of Sciences, Beijing \\ 100049, P. R. China; 'State Key Laboratory of Nonlinear Mechanics, Institute of mechanics, Chinese Academy of Sciences, \\ Beijing 100190, P. R. China and ${ }^{\S}$ Research Center for Green Printing Nanophotonic Materials, Suzhou University of Science \\ and Technology, Suzhou 215009, P. R. China.
}

\section{Supporting Information}

ABSTRACT: Precisely eontrolling control of particles coassembly has attracted great attention for fabricating intricate structures and functional materials. However, achieving precise co-assembly of one-dimensional (1D) binary superstructures remains challenging due to the constrained thermodynamic stability and lack of general strategies to control the 1D ordered arrangement of mixed particles. Here, we propose a facile strategy to achieve programmed co-assembly of $1 \mathrm{D}$ binary superstructures by liquid soft confinement without particles modification or external field. It reveals that binary particles undergo stepwise confinement and programmed co-assembly in the gradually shrinking and spatially tunable liquid soft confinement. Through tuning the liquid confined space and particles composition, diverse 1D binary superstructures with precisely controlled periodicity, orientation and symmetry are achieved, which shows generality for various particles of different sizes and materials. This work provides a promising route to refined patterning and manufacturing complex materials.

Programming particles co-assembly is of scientific significance for exploring new assembly mechanisms, ${ }^{1,2}$ and is of particular interest to bottom-up manufacturing a wide range of intricate structures and complex materials. ${ }^{3,4}$ In the pastOver the past two decades, a large variety of particle-based superstructures with high-long-range order (such as crystalline materials or superlattices) ${ }^{5,6}$ have been achieved by programmed two- or threedimensional co-assembly, driven by entropy-dictated ordering, ${ }^{7,8}$ grafted molecule interactions (such as DNA hybridization), interparticle magnetic forces ${ }^{11}$ - or electrostatic interaction, ${ }^{12}$ and so on. ${ }^{13}$ So farCurrently, programmed 1D co-assembly has been reported by linkers mediated ${ }^{14}$, solvent guided ${ }^{15,16}$ and magnetic field induced $^{17,18}$ assembly approaches based on specific interaction mechanisms. However, these methods require specific particle modification or external field to control the selective and directed interparticle interaction, and the long-range orientation of the $1 \mathrm{D}$ co-assembled superstructures is intrinsically difficult to control. ${ }^{19,20}$ Here, we propose a general and facile strategy to pro- gram the particles co-assembly by liquid soft confinement, and achieve a series of precisely controlled 1D binary superstructures, needless of particle modification or external field.

The strategy presented in this work is based on confined coassembly of two sized particles. Here, we build the gradually shrinking and spatially tunable liquid soft confinement to endow the particles assembly with 'time-_selectivity' and 'space-_selectivity'. As shown in Figure 1a-c, the evaporation mediated rupture of binary particles suspension is controlled by a microfabricated pillars array, ${ }^{21}$ and the yielded resulting capillary bridge provides the-gradually are transported by outward capillary flow. ${ }^{22}$ As-Because the pillar interval of the template influences the formed liquid pattern ${ }_{2}$ resultinged fromthrough competition of the Laplace pressure, ${ }^{23}$ here the vertical interval of the pillars array is designed to be double the horizontal interval (i.e., the distance between two nearest neighboring pillars). Utilizing anionically stabilized polystyrene particles labeled with different fluorescent dyes and a superhydrophilic substrate, direct real-time microscopy analysis reveals the stepwise confinement of binary particles. In the liquid soft confinement, large particles are firstly confined, first and serve as the a secondary template for the following subsequent coassembly with of small particles (Video S1). Besides,Furthermore, the number ratio of small to large particles $\left(N_{\mathrm{S}} / N_{\mathrm{L}}\right)$ influences the time selectivity of the binary particles assembly. When $N_{\mathrm{S}} / N_{\mathrm{L}}$ is approximately $6-8$ (Figure $1 \mathrm{~d}$ and Video S2), small particles are confined to co-assemble with the already assembled large particles. Then, a 'grafting' 1D binary superstructure is formed, in which closely arranged large particles are surrounded by small particles. Given that the particles assembly in the liquid soft confinement proceeds from a unfixed via semifixed to fixed state, when $N_{\mathrm{S}} / N_{\mathrm{L}}$ is increased to aboutapproximately 12-16 (Figure 1e), small particles are confined to coassemble with large particles that are still in semifixed state, followed by an error-and-repair process ${ }^{24}$ (Video S3). Finally, a 'segmented' 1D binary superstructure is formed, in which large particles are evenly spaced by a cluster of small particles. For an intermediate case with $N_{\mathrm{S}} / N_{\mathrm{L}}$ about approximately $8 \sim 10$, a graft- 
ing superstructure is obtained, in which a single particle tocates-is located at the saddle site ${ }^{25}$ between each two large particles (Figure S1). Therefore, the formation of the secondary template, constituted by large particles, overcomes the chaos ${ }^{26}$ in the coassembly process. For a given size ratio of small to large particles $\left(D_{\mathrm{S}} / D_{\mathrm{L}}\right), N_{\mathrm{S}} / N_{\mathrm{L}}$ can be tuned to program the binary particles assembly process to achieve different $1 \mathrm{D}$ co-assembled superstructures. els). Blue arrows indicate the transporting direction of particle dragged by capillary flow. When $N_{\mathrm{S}} / N_{\mathrm{L}}$ is about 6 approximately $\underline{6}-8$, small particles arrange progressively along two contours of the closely arranged large particles, forming the 'grafting' 1D | binary superstructure. When $N_{\mathrm{S}} / N_{\mathrm{L}}$ is approximately $12-16$, small particles first occupy and accumulate at the 2-fold saddle sites, and then arrange along contours of and in the space between large particles, thus generating the 'segmented' $1 \mathrm{D}$ binary superstructure. Scale bars, $3 \mu \mathrm{m}$.

| Besides,-The space -selectivity of binary particles assembly is critical to precise co-assembly of $1 \mathrm{D}$ binary superstructures, and can be programmed by tuning the liquid confined space. Formed between a superhydrophilic template and substrate, the liquid confined space has the shape of a quadrangular column with the waisted cross section. Driven by lowering thedecreasing surface energy, large particles are prior-first confined in the capacious middle region, followed by confined assembly of small particles

| in the residual space. Firstly, Tto control the large particles assembly, the width of liquid confinement $(\mathrm{W})$, which is defined as

the distance between the two three-phase contact line (TCL) of the liquid confinement, is investigated. When $\mathrm{W}>>D_{\mathrm{L}}$, large particles would assemble into multi-particle chains ${ }^{21,23}$; when $\mathrm{W}<D_{\mathrm{L}}$, large particles would beare prevented from migrating into the liquid confined space, thus failing the to achieve $1 \mathrm{D}$ assembly. By Using a square-pillar-template with $\mathrm{H}$ of $5.0 \mu \mathrm{m}$, programmed coassembly from binary particles with diameters of $3.00 \mu \mathrm{m}$ and $0.34 \mu \mathrm{m}$ is achieved (Figure 2a and 2b, Figure S2 and Video S4). By measuring $\mathrm{W}$ by for the templates with different pillar heights of $1.7 \mu \mathrm{m}, 3.0 \mu \mathrm{m}, 5.0 \mu \mathrm{m}$ and $7.0 \mu \mathrm{m}$, it is found that $\mathrm{W}$ increases with $\mathrm{H}$, which is adjustable for the assembly of particles with different sizes (Figure 2c). For another case when $\mathrm{W}$ is slightly larger than $D_{\mathrm{L}}$ (Figure $\mathrm{S} 3$ ), typical grafting or segmented 1D binary superstructures are not formed, but another segmented superstructure is obtained. The large particles in the limited confined space tend to be fixed ${ }^{27}$ and the spacing between large particles is mainly-primarily influenced by the local number of large particles, nearly independent of the small particles. Subsequently, small particles are confined to co-assemble with the fixed large particles to form another segmented superstructure. Generally, the space selectivity of large particle assembly can be programmed by controlling $\mathrm{W}$ through tailoring $\mathrm{H}$. ThenMoreover, to program the space selectivity of the small particles assembly, the residual space excluding large particles in the liquid confinement can be controlled by tuning the receding angle of the substrate $\left(\theta_{R}\right)$. Critical width of liquid confinement when large particles is confined decreases with $\theta_{R}{ }^{28}$ leading to reduced space to accommodate small particles, thus the 1D binary superstructures is-are formed with decreased (Figure 2d and 2e) or none loading of small particles (Figure S4). With the a hydrophobic substrate, the programmed co-assembly is frustrated due to the-instability of the liquid confinement. It should be notediced that the vacancy near the TCL (dotted framed region in Figure 2d) is also influenced by $D_{\mathrm{L}}$ under-below a certain pillar height, thus the range of $D_{\mathrm{S}} / D_{\mathrm{L}}$ is limited for programmed co-assembly. A series of experiments with different $D_{\mathrm{S}} / D_{\mathrm{L}}$ values are-were conducted, and it is found thatdetermined that $-D_{\mathrm{S}} / D_{\mathrm{L}}$ should be smaller than 0.5 to achieve well controlledwell-controlled co-assembly (Figure S5). When $D_{\mathrm{S}} / D_{\mathrm{L}}$ is larger than 0.5 , the vacancy near the TCL is not large enough to match the size of the small particles, and the programmed co-assembly fails (Figure S6). Besides microscale particles, nanoparticles co-assembly can also be programmed to achieve a variety of well-defined 1D binary superstructures (Figure S7) by synthetically tuning the pillar height, receding angle of the substrate and particles composition. To sum up, programmed co-assembly of various $1 \mathrm{D}$ binary superstructures can be achieved by tuning the liquid confined space and particles composition. 


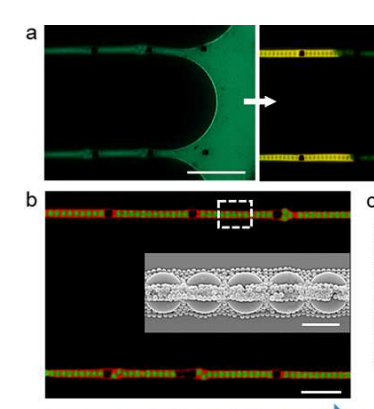

d Increase the receding angle of substrate
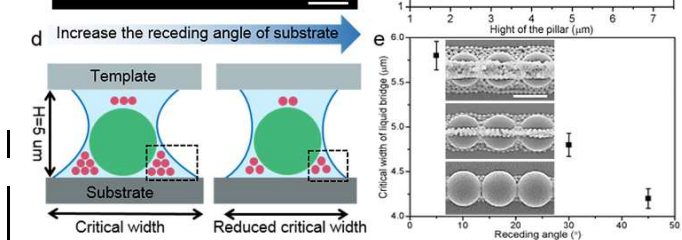

Figure 2. Programmed co-assembly by controlling the liquid confined space. (a) The pProgrammed co-assembly process shows that the aligned large particles serve as the secondary template for following the subsequent co-assembly with-of small particles. The arrows indicate the time sequence $;$ s $s$ cale bar, 50 $\mu \mathrm{m}$. (b) Bottom-view confocal micrograph of the $1 \mathrm{D}$ binary superstructures array (Scale bar, $20 \mu \mathrm{m}$ ) and magnified SEM image (Scale bar, $5 \mu \mathrm{m}$ ) of the boxed region. (c) Experimentally measured width of liquid confinement increases with the pillar height, indicating that the pillar height can be adjusted to control the assembly of large particles with different sizes. (d) Schematic for the cross section of binary particles in the liquid confinement, illustrating that the number of small particles accommodated in the vacancy near the TCL (dotted framed region) increases with the critical width when the large particle is confined. (e) Employing the-substrates with different receding angles $\left(\theta_{R}\right)$ of $5^{\circ}, 30^{\circ}$ and $45^{\circ}$, the measured critical widths and the corresponding SEM images of the resulted-resulting 1D binary superstructures, showing show that the critical width decreases with $\theta_{R}$, leading to different co-assembled superstructures with reduced loading of small particles. Scale bars, $3 \mu \mathrm{m}$.

Furthermore, the orientation of the 1D binary co-assembled superstructures can be programmed to achieve different patterns. Induced by a straight stripe- or curve stripe-pillar-template, different liquid patterns can be formed in the gap between the pillars top and substrate $^{29}$ (top-view SEM images of the pillartemplates and the scheme for control of the gap are shown in Figure S8). In the liquid soft confinement, the particles moving trajectory is controlled, and the elongation of the assembled region can be directed to form different patterns. Thus, well controlled co-assemblies can be coupled into designed routings, and diverse patterns are achieved, such as straight-line patterns, curve patterns or compound patterns consisting of lines and curves (Figure 3a and $3 \mathrm{c}$, and Video S5). Based on the analysis above, by enlarging the liquid confined space $3 b$ ), leads large particles to stack in two layers in the formed-resulting 1D binary superstructure, and the number of large particle chains in each layer is-exhibitsin an arithmetic progression (Figure $3 \mathrm{~d}$ and $3 \mathrm{e}$ ). Therefore, the orientation and configuration of the 1D binary superstructures can be programmed to achieve different patterns.
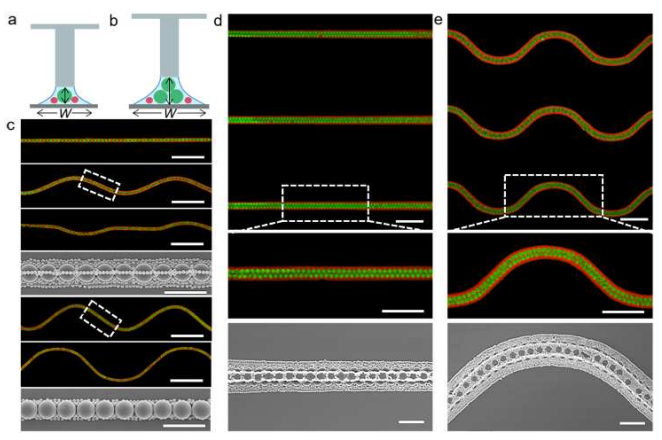

Figure 3. Programmed orientation of the 1D binary superstructures to achieve different patterns. $(a, b)$ Schematic illustrations for the cross-section of the liquid confined system formed in the gap between a hydrophilic substrate and the pillar top of a straight stripe- or curve stripe-pillar-template, showing that the width of liquid confinement, $-\mathrm{W}_{2}$ is increased when the gap is enlarged, and large particles stack in more than one layer. (c) Corresponding to the schematic sketch in (a), 1D binary superstructures, with well controlled orientation, are achieved. (d, e) Corresponding to the schematic sketch in (b), arrays of straight or curved superstructures with large particles stacking in two layers, are achieved. The number of large particle chains in the upper layer is less than that in the bottom layer. Scale bars are, $20 \mu \mathrm{m}$ in the confocal micrographs and $5 \mu \mathrm{m}$ in the SEM images.

Besides-In addition to symmetric 1D binary superstructures, programmed co-assembly of asymmetric 1D binary superstructures is also achieved, and the correlation between the width of the liquid confinement $W^{\prime}$ (marked in Figure 4a) and the pillar height $\mathrm{H}$ is quantified experimentally and theoretically. With the pillar top of a stripe-pillar-template closely contacting a superhydrophilic substrate, particles dragged by outward capillary flow are blocked to gather by one side of a pillar (Figure 4a). With the evaporation of solvent, the formed liquid confinement is divided by the stripe-pillar into two parts. In the liquid soft confinement at the particles' gathering side, programmed co-assembly occurs with small particles asymmetrically arranging along one-side of the aligned large particles' contour near the TCL (Video S6). Moreover, we find a linear increase of the measured $\mathrm{W}^{\prime}$ dependent on $\mathrm{H}$, with the-a slope of about-approximately 0.4 (Figure 4b), which is further supported by the theoretical analysis (Supplementary Discussion). The linear dependence of $\mathrm{W}^{\prime}$ on $\mathrm{H}$ offers the quantitative basis for particles assembly by controlling the liquid confined space through tailoring the pillar height. Accordingly, by utilizing the - template with the pillar height of $18.0 \mu \mathrm{m}$, asymmetric 1D binary superstructures, constituted by binary particles with diameters of $3.00 \mu \mathrm{m}$ and $1.00 \mu \mathrm{m}$, are achieved (Figure $4 \mathrm{c}$, d). The co-assembled superstructures can be diversified by intentionally adjusting the $N_{\mathrm{S}} / N_{\mathrm{L}}$ and $\theta_{R}$ (Figure S9a). By decreasing the pillar height, diverse $1 \mathrm{D}$ binary superstructures assembled by particles with the size from micrometer (Figure S10) to nanometer (Figure S9b) can be achieved, and these ordered structures can be well controlled in the long range (Figure S11). SignificantlyPromisingly, this facile strategy shows generality for particles-assembly of various materials, such as inorganic particles, metal particles, or mixed particles with different materials (Figure S12).
Formatted: Font color: Custom Color(RGB $(56,86,35))$ 


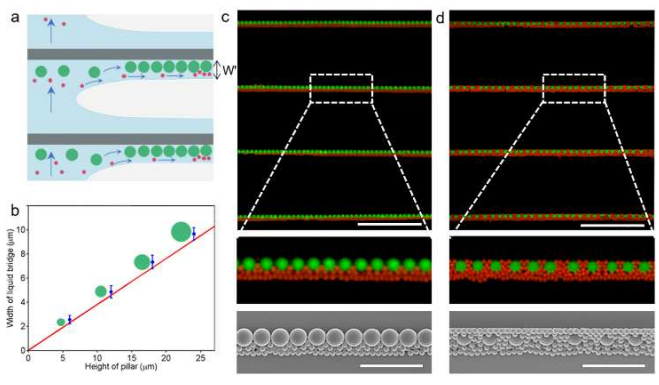

Figure 4. Programmed co-assembly of asymmetric 1D binary superstructures and quantified correlation between width of liquid confinement $\left(\mathbf{W}^{\prime}\right)$ and pillar height. (a) Particles gather

by one side of the straight stripe-pillars (greygray), the top of which closely contacts the superhydrophilic substrate. The liquid confinement is divided into two parts, in one of which small particles asymmetrically arrange along one-side of the contour of the aligned large particles' contour near the TCL. Blue arrows indicate the transperting transport direction of particles. (b) Experimental values (blue dots with error bars) and theoretical values (red line) of $\mathrm{W}^{\prime}$ demonstrate a linear increase with the pillar height, which is adjustable to control large particle assembly. (c, d) Bottom-view confocal micrographs with the magnified view of the boxed region and top-view SEM images show the asymmetric 1D binary superstructures, including grafting (c) and segmented (d) superstructures. Scale bars are- $50 \mu \mathrm{m}$ in the confocal micrographs and $10 \mu \mathrm{m}$ in the SEM images.

In conclusion, distinct from the specific interparticle interaction mechanisms that enable programmed two- or three-dimensional co-assembly, we present a general and facile strategy by liquid soft confinement to achieve programmed co-assembly of 1D binary superstructures. Through tuning the liquid confined space and particles composition, the binary particles assembly can be programmed with relatively independent control. Accordingly, a wide range of 1D binary superstructures, with precisely controlled periodicity, orientation and symmetry, are achieved. Even freestanding particle chains can be foreseen by a fluidic device. Given that this programmed co-assembly strategy is applicable to differ-

I ent particles combinations, developing multifunctional materials ${ }^{30}$ organized by multicomponent elements with unique plasmonic, magnetic, or optical properties is anticipated.

\section{ASSOCIATED CONTENT}

\section{Supporting Information}

Materials and Methods, Supplementary Discussion, Supplementary Figures, Captions for Supplementary Videos, Supplementary Table, References, and Videos S1 to S6.

\section{AUTHOR INFORMATION}

\section{Corresponding Author}

*ylsong@iccas.ac.cn

\section{Notes}

The authors declare no competing financial interests.

\section{ACKNOWLEDGMENT}

The authors thank the financial support from the National Nature Science Foundation (Grant Nos. 51473173, 21522308, 51573192 and 11572335), the 973 Program (2013CB933004) and the Strategic Priority Research Program of the Chinese Academy of Sciences (Grant No. XDA09020000) and (Grant No. XDB22040403). See the supplementary materials for additional data.

\section{REFERENCES}

(1) Wang, L.; Xu, L.; Kuang, H.; Xu, C.; Kotov, N. A. Acc. Chem. Res. 2012, 45, 1916-1926.

(2) Jones, M. R.; Seeman, N. C.; Mirkin, C. A. Science 2015, 347, 1260901.

(3) Li, F.; Josephson, D. P.; Stein, A. Angew. Chem. Int. Ed. 2011, 50, 360-388.

(4) Wei, J.; Schaeffer, N.; Pileni, M.-P. J. Am. Chem. Soc. 2015, 137, 14773-14784.

(5) Dong, A. G.; Chen, J.; Vora, P. M.; Kikkawa, J. M.; Murray, C. B. Nature 2010, 466, 474-477.

(6) Macfarlane, R. J.; Lee, B.; Jones, M. R.; Harris, N.; Schatz, G. C.; Mirkin, C. A. Science 2011, 334, 204-208.

(7) Paik, T.; Diroll, B. T.; Kagan, C. R.; Murray, C. B. J. Am. Chem. Soc. 2015, 137, 6662-6669.

(8) Boles, M. A.; Talapin, D. V. J. Am. Chem. Soc. 2015, 137, 4494-4502. (9) Wang, Y.; Wang, Y.; Zheng, X.; Ducrot, É.; Lee, M.-G.; Yi, G.-R.; Weck, M.; Pine, D. J. J. Am. Chem. Soc. 2015, 137, 10760-10766. (10) Rogers, W. B.; Manoharan, V. N. Science 2015, 347, 639-642. (11) Yang, Z.; Wei, J.; Bonville, P.; Pileni, M.-P. J. Am. Chem. Soc. 2015, $137,4487-4493$

(12) Kostiainen, M. A.; Hiekkataipale, P.; Laiho, A.; Lemieux, V.; Seitsonen, J.; Ruokolainen, J.; Ceci, P. Nat. Nanotechnol. 2013, 8, 52-56. (13) Künzle, M.; Eckert, T.; Beck, T. J. Am. Chem. Soc. 2016, 138, 12731-12734.

(14) Wang, Y.; Wang, Y.; Breed, D. R.; Manoharan, V. N.; Feng, L.; Hollingsworth, A. D.; Weck, M.; Pine, D. J. Nature 2012, 491, 51-55.

(15) Gröschel, Y.; Walther, A.; Löbling, T. I.; Schacher, F. H.; Schmalz, H.; Müller, A. E. Nature 2013, 503, 247-251.

(16) Qiu, H.; Hudson, Z. M.; Winnik, M. A.; Manners, I. Science 2015, 347, 1329-1332.

(17) Khalil, K. S.; Sagastegui, A.; LI, Y.; Tahir, M. A.; Socolar, J. S.; Wiley, B. J.; Yellen, B. B. Nat. Commun. 2012, 3:794.

(18) Bannwarth, M. B.; Utech, S.; Ebert, S.; Weitz, D. A.; Crespy, D.; Landfester, K. ACS Nano 2015, 9, 2720-2728.

(19) Tang, Z.; Kotov, N. A. Adv. Mater. 2005, 17, 951-962.

(20) Cademartiri, L.; Bishop, K. J. Nat. Mater. 2015, 14, 2-9.

(21) Su, B.; Zhang, C.; Chen, S.; Zhang, X. Y.; Chen, L.; Wu, Y.; Nie, Y.; Kan, X.; Song, Y.; Jiang, L. Adv. Mater. 2014, 26, 2501-2507.

(22) Deegan, R. D.; Bakajin, O.; Dupont, T. F.; Huber, G.; Nagel, S. R.; Witten, T. A. Nature 1997, 389, 827-829.

(23) Huang, Z.; Su, M.; Yang, Q.; Li, Z.; Chen, S.; Li, Y.; Zhou, X.; Li, F.; Song, Y. Nat. Commun. 2017, 8:14110.

(24) Nagao, K.; Inuzuka, T.; Nishimoto, K.; Edagawa, K. Phys. Rev. Lett. 2015, 115, 075501 .

(25) Velikov, K. P.; Christova, C. G.; Dullens, R. P.; van Blaaderen, A. Science 2002, 296, 106-109.

(26) Chen, M.; Cölfen, H.; Polarz, S. ACS Nano 2015, 9, 6944-6950

(27) Yamaki, M.; Higo, J.; Nagayama, K. Langmuir 1995, 11, 2975-2978. (28) Kuang, M.; Wang, J.; Bao, B.; Li, F.; Wang, L.; Jiang, L.; Song, Y. Adv. Opt. Mater. 2014, 2, 34-38.

(29) Guo, D.; Li, C.; Wang, Y.; Li, Y.; Song, Y. Angew. Chem. Int. Ed. 2017, 56, 1-6.

(30) Boles, M. A.; Engel, M.; Talapin, D. V. Chem. Rev. 2016, 116, 11220-11289. 
10

11

12

13

14

15

16

17

Insert Table of Contents artwork here

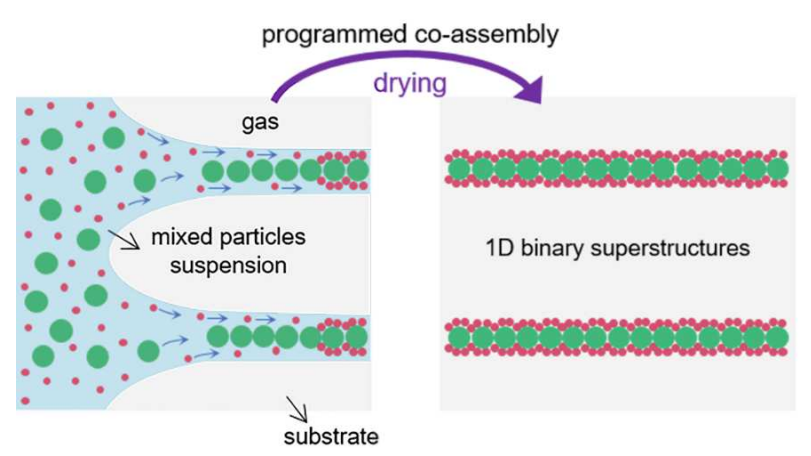

